\title{
Focus on regenerative medicine
}

Regenerative medicine encompasses a broad array of therapies, from hematopoietic stem cell therapies currently in clinical practice to therapies derived from human embryonic stem cells and human induced pluripotent stem (iPS) cells. Ten cell therapies are currently on the US market, ranging from treatments for hematopoietic reconstitution (e.g., Hemacord and Ducord), to mucogingival wounds (Gintuit) to cartilage repair (Carticel), all the way to cosmetics for the correction of severe wrinkles (LaVive).

More than most other fields of biomedicine, cell therapies face a complex path to market. Even the scale-up of less controversial adult stem cell therapies requires innovative thinking and new delivery systems (Commentary, p. 729). Developing therapies derived from pluripotent stem cells presents even stiffer challenges, although several groups are pushing treatments into the clinic for such conditions as age-related macular degeneration, refractory thrombocytopenia, epidermolysis bullosa (Nat. Biotechnol. 31, 483-486, 2014) and diabetes. Translation of regenerative medicine treatments involves not only regulatory issues (see

Podcast; Correspondence p. 721) but also ethical, political (Correspondence, p. 724) and legal (Patent Article, p. 742; Patent Table, p. 749) issues. In the face of these crosswinds, several states have set up specific programs to facilitate commercialization of cell therapy products (Feature p. 736).

In the research arena, progress is accelerating through advances at the intersection of cell biology and technology. A key aspect of any allogeneic cell therapy is the issue of immune rejection of transplanted cells. Decades of clinical experience in transplanting solid organs and hematopoietic cells offer important lessons on immunosuppression, and new tolerance-induction strategies are being developed specifically for applications in cell therapy (Review, p. 786). The ability to improve cell therapies depends on understanding the fate of cells after transplantation into patients. Frank, Murry and colleagues review the capabilities and limitations of clinical imaging technologies for assessing cell fate and the extent of regeneration (Review, p. 804). Conventional transplantation of whole organs is easier on sensitive cells than cell therapy because the cells' native microenvironment is preserved. 3D bioprinting, a high-tech version of traditional tissue engineering, aims to produce transplantable tissues and organs in the laboratory to address the severe shortage of donor organs (Review, p. 773; News Feature, p. 716).

For some diseases, it may be possible to avoid cell therapy altogether using small molecules, proteins or biomaterials to activate latent molecular pathways of regeneration or to introduce new ones. Watt and colleagues consider the prospects for enhancing regeneration by manipulating the stem-cell niche (Review, p. 795).

Innovations in in vitro modeling of development and disease are facilitating the study of tissue regeneration and the search for drugs to control these processes. Microfluidic 'organs-on-chips' allow more precise control over cell patterning and microenvironmental conditions than conventional cell-culture systems and show promise for recapitulating organ-level functions. Potential applications in regenerative medicine include drug testing and investigation of organ development, physiology and pathology (Perspective, p. 760). In a separate piece, Ken Garber explores the use of patient-derived iPS cell lines as a tool for creating neuron- and glia-like cells to probe disease mechanisms and screen drug candidates (News Feature, p. 712). An editorial discusses the importance of a broad range of different treatment modalities in realizing the potential of regenerative medicine (Editorial, p. 699).

$K A \& A M$

Written by Kathy Aschheim \& Andrew Marshall

\section{More online....in a joint focus with Nature Medicine}

Readers online will find more content related to regenerative medicine in a joint web focus with Nature Medicine (http://www.nature.com/focus/regenerative_medicine). As well as additional News and Feature content, several Reviews and a Perspective are featured:

- Stefanie Dimmeler, Sheng Ding, Thomas Rando and Alan Trounson outline the challenges in translating knowledge of stem cell biology into regenerative medicines and discuss strategies for overcoming them.

- Darrell Kotton and Edward Morrisey review emerging techniques that allow the identification of putative stem and progenitor cells in the lung and summarize current understanding of the molecular mechanisms regulating lung development and regeneration that may lead to therapeutic application.

- Ya-Chieh Hsu, Lishi Li and Elaine Fuchs discuss the different stem cell populations in the skin required to maintain its function, how this organ can serve as a model for studying the interactions of stem cells with their microenvironment/niche and the implications for therapeutic applications.

- Avital Mendelson and Paul S. Frenette discuss the cellular components of the hematopoietic stem cell niche and the mechanisms by which these regulate the differentiation and maintenance of hematopoietic stem cells.

- Juhyun Oh, Yang David Lee and Amy Wagers overview the mechanisms leading to age-dependent decline in stem cell function and potential therapeutic avenues for reversal of these effects.

- Stuart Forbes and Nadia Rosenthal explore our current understanding of the importance of the local microenvironment in determining the success of exogenous and endogenous stem cells in repairing damage.

Hannah Stower, Associate Editor, Nature Medicine

\section{Focus Issue Podcast}

\section{Anthony Davies}

Anthony Davies has worked in the cell and gene therapy field for $\sim 20$ years, at such companies as Onyx Pharmaceuticals and Geron. He is now president of Dark Horse Consulting. Nature Biotechnology

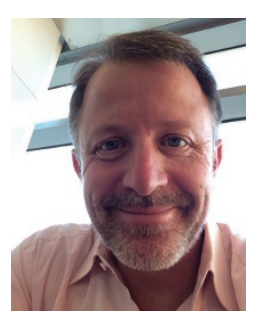
spoke to Davies about the history of human embryonic stem cells in clinical trials, challenges in bringing future products to market and the overall prognosis for the field. http://www. nature. com/nbt/podcast/index.html. 